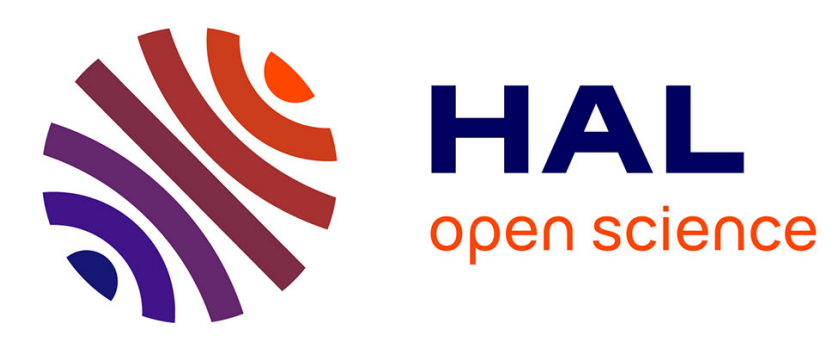

\title{
Liénard systems and potential-Hamiltonian decomposition II - Algorithm
}

Jacques Demongeot, Nicolas Glade, Loïc Forest

\section{To cite this version:}

Jacques Demongeot, Nicolas Glade, Loïc Forest. Liénard systems and potential-Hamiltonian decomposition II - Algorithm. Comptes rendus de l'Académie des sciences. Série I, Mathématique, 2007, 344, pp 191-194. hal-00211817

\section{HAL Id: hal-00211817 https://hal.science/hal-00211817}

Submitted on 23 Jan 2008

HAL is a multi-disciplinary open access archive for the deposit and dissemination of scientific research documents, whether they are published or not. The documents may come from teaching and research institutions in France or abroad, or from public or private research centers.
L'archive ouverte pluridisciplinaire HAL, est destinée au dépôt et à la diffusion de documents scientifiques de niveau recherche, publiés ou non, émanant des établissements d'enseignement et de recherche français ou étrangers, des laboratoires publics ou privés. 


\title{
Systèmes de Liénard et décomposition potentielle-Hamiltonienne II - Algorithme
}

\author{
Liénard systems and potential-Hamiltonian decomposition II - \\ Algorithm \\ Jacques DEMONGEOT ${ }^{\mathrm{a}, \mathrm{b}}$ Nicolas GLADE $^{\mathrm{b}}$ Loic FOREST $^{\mathrm{b}}$ \\ ${ }^{\text {a }}$ Institut Universitaire de France \\ b TIMC-IMAG UMR CNRS 5525 \\ University J. Fourier Grenoble \\ Faculty of Medicine 38700 La Tronche France \\ Tel. 0456520108 \\ FAX 0476768844
}

\begin{abstract}
We show here how to approach with an increasing precision the limit-cycles of Liénard systems, bifurcating from a stable stationary state, by contour lines of Hamiltonian systems derived from a potential-Hamiltonian decomposition of the Liénard flow. We evoked in a previous Note the case (non polynomial) of pure potential systems (n-switches) and pure Hamiltonian systems (2D Lotka-Volterra), and here we show that, with the proposed approximation, we can deal with the case of mixed systems (van der Pol or FitzHugh-Nagumo) frequently used for modelling oscillatory systems in biology. We suggest finally that the proposed algorithm, generic for $\mathrm{PH}-$ decomposition, can be used for estimating the isochronal fibration in some specific cases near the pure potential or Hamiltonian systems. In a following Note, we will give applications in biology of the potential-Hamiltonian decomposition.

\section{Résumé}

Nous montrons ici comment approcher, avec une précision croissante, les cycles limites des systèmes de Liénard par les courbes de niveau du système Hamiltonien obtenu à partir d'une décomposition potentielle-Hamiltonienne. Nous avons présenté, dans une Note précédente, des cas non polynomiaux de systèmes purement potentiels (type n-switch) et purement Hamiltoniens (type Lotka-Volterra 2D), puis nous donnons ici des exemples de décomposition potentielle-Hamiltonienne pour des systèmes de type van der Pol (mixtes) qui sont d'usage fréquent en modélisation des systèmes biologiques oscillants. Nous suggérons enfin que l'algorithme proposé, générique pour la décomposition potentielle-Hamiltonienne, soit utilisé pour estimer la fibration isochrone, dans le cas de systèmes voisins des systèmes purs (potentiels ou Hamiltoniens). Dans une Note future, nous décrirons des applications biologiques précises de la décomposition potentielle-Hamiltonienne.
\end{abstract}

\section{Estimation of the limit-cycle of a Liénard system}

Liénard systems [1] are 2-dimensional ordinary differential equations (2D ODEs) defined by $d x / d t=y$, $d y / d t=-g(x)+y f(x)$, where $g$ and $f$ are polynomials. Liénard systems have in general as asymptotics a unique limit-cycle [2]. The existence of such a periodic orbit explains that the use of Liénard systems is

Email addresses: jacques.demongeot@imag.fr (Jacques DEMONGEOT), nicolas.glade@imag.fr (Nicolas GLADE), loic.forest@imag.fr (Loic FOREST). 
universal (especially in biological, electrical, mechanical,..., modeling of oscillatory systems).

Algebraic estimation of the limit-cycles for the Liénard systems has already been studied by searching closed polynomial curves passing through some of their points [3]. They are trajectories of Hamiltonian systems, but it has not been proposed before to extend the estimation of the dynamical flow to the whole state space by adding a potential part. The PH-decomposition of Liénard systems we propose here could help also, as noticed in [3], to clarify the relationship between positive loops of their Jacobian matrix and the number of their attractors $[4,5]$. This problem is an open mathematical question of great interest in biology, partially solved for discrete systems [6-9]. Many differential systems of dimension 2 can be reduced into Liénard systems, which increases the interest of the PH-decomposition. For example, the FitzHugh-Nagumo system [10] defined by the following equations, where $p$ is a cubic polynomial:

$$
d x / d t=y+p(x), \quad d y / d t=c x-b y+a
$$

becomes the following Liénard system by changing $y$ into $Y=y+p(x)$ :

$$
d x / d t=Y, \quad d Y / d t=[c x-b p(x)+a]+Y[p(x)-b]
$$

i.e. a Liénard system called van der Pol system, if $p(x)=\mu x\left(1-x^{2} / 3\right), \mathrm{c}=-1, \mathrm{a}=\mathrm{b}=0$. Then to approach algebraically its limit-cycle, we use the PH-decomposition proposed in Theorem 1 of [1], by remarking that:

$$
f_{1}^{[1](0)}(x, y)=\sum_{i>j} a_{i j} \frac{x^{i+1} y^{j}}{i+1}+\sum_{i=j} a_{i j} \frac{x^{i+1} y^{j}}{2(i+1)}, \quad g_{1}^{[0](1)}(x, y)=\sum_{i>j} j b_{i j} x^{i} y^{j-1}+\sum_{i=j} j b_{i j} \frac{x^{i} y^{j-1}}{2}
$$

We impose that (i) the potential and Hamiltonian flows are orthogonal in each point of the phase plane, (ii) a Hamiltonian trajectory passes through points of the limit-cycle $M_{1}, M_{2}, M_{3}, \ldots, M_{K(n, m, p)}$ numerically estimated, (iii) the gradient of $\mathrm{P}$ vanishes in $M_{i}$ 's points. Then we identify the coefficients of the polynomials appearing on left and right hand sides of the orthogonality equation. $K(n, m, p)$ is chosen in order to identify all non-zero coefficients of polynomials $\mathrm{P}$ and $\mathrm{H}$, taking into account the fact that orthogonality equations give already equalities between coefficients of $f_{i}^{[k](l)}$ 's and $g_{i}^{[k](l)}$ 's like:

$$
\forall i, j \geq 2, \quad a_{i j}=-\frac{(i+1)(i+2)}{j(j-1)} a_{i+2, j-2}
$$

Then we search for the level of the contour line $C_{M}$ of the Hamiltonian surface passing through the set M of points $M_{i}$ chosen on the limit-cycle (cf. Fig. 1 Left). As $m$ and $p$ tend to infinity, $K(n, m, p)$ tends to infinity and if we progressively choose the points $M_{i}$ more and more dense on the limit-cycle, $C_{M}$ tends to this limit-cycle.
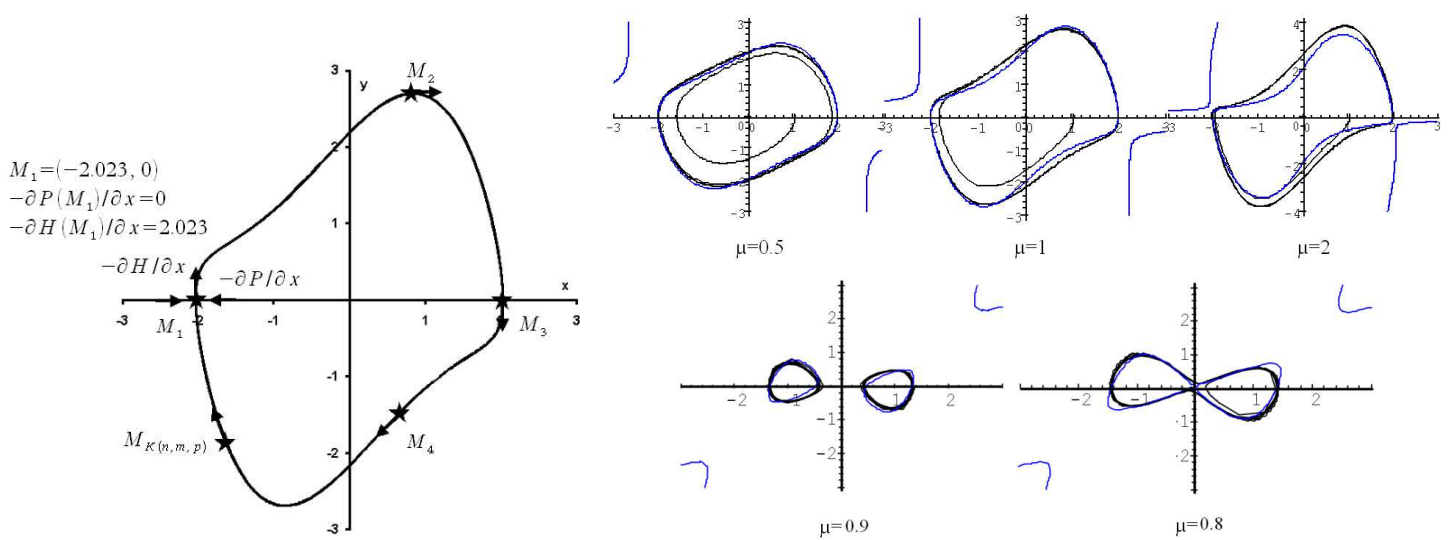

Figure 1. - (Left) Algebraic approximation of the van der Pol limit-cycle. Local PH-decomposition at the points $M_{1}, M_{2}, M_{3}$, $M_{4}, \ldots, M_{K(n, m, p)}$ of the van der Pol limit-cycle. (Right) PH-decompositions (Up) for the van der Pol system and (Down) for an "lemniscate" system.

Let's consider now the van der Pol system defined by $d x / d t=y, d y / d t=-x+\mu\left(1-x^{2}\right)$. Its Hamiltonian part given by the PH-decomposition proposed in [1] is: $H(x, y)=\left(x^{2}+y^{2}\right) / 2-3 \mu x y / 2+3 \mu y x^{3} / 8-\mu x y^{3} / 24-$ 2.023 and contour lines passing through $M_{1}$ (and $M_{3}$ ) are obtained by choosing the level 0 for $\mathrm{H}$ (of fig.1 up).

The "lemniscate" system has been proposed by R. Thom [11] to model the amphibian embryogenesis:

$$
d x / d t=y, \quad d y / d t=x\left(1-x^{2}\right)-y\left(\mu-x^{2}\right)
$$


Hamiltonian parts for $\mu=0.8$ and $\mu=0.9$ are respectively :

$H_{0.8}(x, y)=-\left(x^{2}-y^{2}\right) / 2+x y / 2+x^{4} / 4+x y^{3} / 24-3 y x^{3} / 8$ and $H_{0.9}(x, y)=H_{0.8}(x, y)+0.1$

and the contour lines are obtained by choosing the level $H_{\mu}(x, y)=0$ for $\mu=0.8$ and 0.9 (fig. 1 down).

Examples of potential-Hamiltonian decomposition given above generalize the results given in [12]. More generally, two factors could favour the resolving of the problem which consists in counting attractors of Liénard systems, which is a part of the historical XVI ${ }^{\text {th }}$ Hilbert problem [13]: (i) Lyapunov functions found in differential systems (e.g. Dolbeault techniques [14]) and (ii) recent results obtained for discrete systems [6-9] could give ideas of proofs, especially if the differential systems can be approached by discrete automata (like Wilson-Cowan systems by Hopfield like networks [15]).

\section{PH-decomposition and isochrons}

In addition to the algebraic estimation of the limit-cycles, the main interest of the decomposition above is to divide the parameters that appear in the right hand side part of the Liénard equations into two sets: - the set of parameters of $\mathrm{P}$, more amplitude controlling - the set of parameters of $\mathrm{H}$, more frequency controlling in the neighbourhood of a limit-cycle of the Liénard system.

The explanation of the separated roles of the parameters is of important biological relevance to explain amplitude or frequency modulation abilities of both genetic and metabolic regulatory systems. This is particularly useful when considering the isochronal fibration [16] transverse to the trajectories, whose analysis allows to predict the entrainment of a biological oscillator by an external periodic signal or its response to an instantaneous stimulation $[17,18]$. In some cases, the isochronal fibration is just made of the orbit networks of the potential part in the PH-decomposition. Let's consider for example the case of an anharmonic oscillator having the unity circle as limit-cycle:

$$
d x / d t=y+x\left(1-x^{2}-y^{2}\right), \quad d y / d t=-x+y\left(1-x^{2}-y^{2}\right)
$$

whose solutions are: $x(t)=-\rho_{0} e^{t} \cos \left(t-\theta_{0}\right) / \sqrt{1+\rho_{0}^{2}\left(e^{2 t}-1\right)}, y(t)=\rho_{0} e^{t} \sin \left(t-\theta_{0}\right) / \sqrt{1+\rho_{0}^{2}\left(e^{2 t}-1\right)}$ Then the potential-Hamiltonian decomposition gives: $H(x, y)=\left(x^{2}+y^{2}\right) / 2$ and $P(x, y)=-\left(x^{2}+y^{2}\right) / 2+\left(x^{4}+y^{4}\right) / 4+x^{2} y^{2} / 2$

It is easy to check that isochrons are just radial orbits of the potential part (cf. fig. 2 (left) and (middle)).
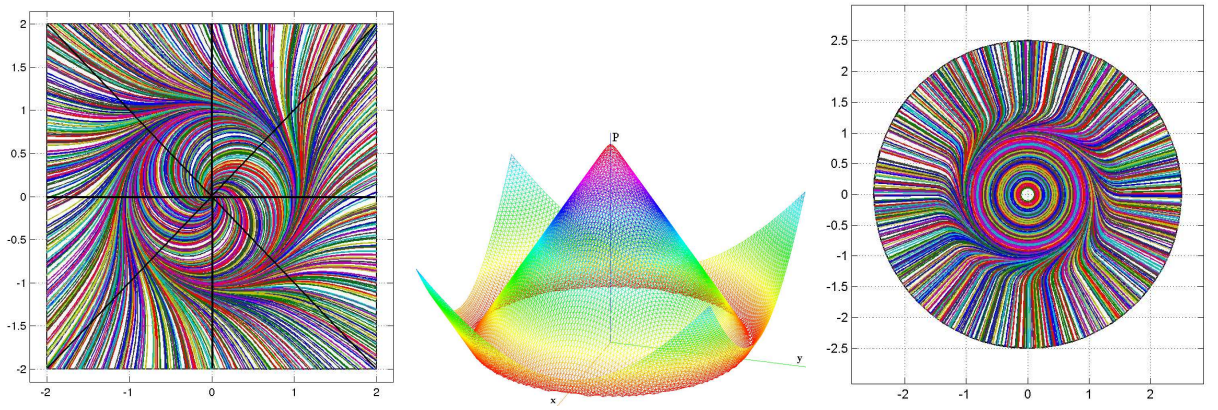

Figure 2. - (left) Phase portrait of the anharmonic oscillator flow (eq. 6) and its isochronal fibration in black. (middle) Representation of the potential $\mathrm{P}$ for the anharmonic oscillator (eq. 6). (right) Example of the differential system (eq. 7) exhibiting in its phase plane a potential behavior on the external border and a Hamiltonian behavior inside the unity circle.

We conjecture this result still holds in the neighbourhood of limit-cycles of a large class of perturbations of such an anharmonic oscillator. This example also shows that a simple inversion of $\mathrm{P}$ and $\mathrm{H}$ is unable to generate a flow giving isochrons as trajectories, but this property holds here in the neighbourhood of the limit-cycle and it is also the case in the neighbourhood of the frontiers of the basin of attraction (as predicted in [17]) for the following system defined on $[1,2.5] \mathrm{x}[0,2 \pi[$, purely potential in its border region:

$$
\frac{d \theta}{d t}=\sup (0,(1.5-\rho)), \quad \frac{d \rho}{d t}=\left\{\begin{array}{l}
1-\rho \text { if } 1 \leq \rho \leq 3 / 2 \\
-(5 / 2-\rho) / 2 \text { if } 3 / 2 \leq \rho \leq 5 / 2
\end{array}\right.
$$

This example shows that the isochronal fibration tends to adopt the Hamiltonian (resp. the potential) flow shape, when the potential (resp. the Hamiltonian) is dominant, which gives to the PH-decomposition another practical use (cf. fig. 2 (right)), especially if the systems are near either pure potential or pure Hamiltonian systems. 


\section{Conclusion}

In this article, we have shown the possibility to explicitely (i.e. by using a precise algorithm) decompose any sufficiently regular 2D-differential system into two parts : a potential one having a dissipative gradient-like behaviour responsible, in general, for the amplitude of a periodic signal generated by the system, and another one, Hamiltonian, expressing the conservative part of the flow responsible for the frequency of this periodic signal. This decomposition can be crucial in applications, particularly in biology, in which we have to detect the parameters responsible respectively for the amplitude and frequency modulation of the signaling. Such parameters are often very difficult to extract and identify, but are necessary for understanding signaling and regulatory mechanisms. The proposed algorithm allows it, and moreover permits to estimate, near both pure potential and Hamiltonian systems, the isochronal fibration associated to the stability basin of a limit-cycle, which is always crucial for biological periodic systems [16-18].

\section{Acknowledgements}

We are very indebted to R. Thom (died in October 2002, formerly President of the French Society for Theoretical Biology) for opening the new field of modeling biological systems with a qualitative approach using singularity theory, and to J.P. Françoise for stimulating discussions and helpful suggestions.

\section{References}

[1] Demongeot J., Glade N., Forest L., Liénard systems and potential-Hamiltonian decomposition I - Methodology, C. R. Acad. Sci. Mathématiques (in press).

[2] Timoteo Carletti T., Villari G., A note on existence and uniqueness of limit-cycles for Liénard systems, J. Math. Anal. Appl. 307 (2005) 763-773.

[3] Giacomini H., Neukirch S., Algebraic approximations to bifurcation curves of limit-cycles for the Liénard equation, Phys. Lett. A 244 (1998) 53-58.

[4] Cinquin O., Demongeot J., Positive and negative feedback : striking a balance between necessary antagonists, J. Theoret. Biol. 216 (2002) 229-241.

[5] Cinquin O., Demongeot J., Positive and negative feedback: mending the ways of sloppy systems, C. R. Acad. Sci. Biologies 325 (2002) 1085-1095.

[6] Aracena J., Ben Lamine S., Mermet M.A., Cohen O., Demongeot J., Mathematical modelling in genetic networks: relationships between the genetic expression and both chromosomic breakage and positive circuits, IEEE Trans. Systems Man Cyber. 33 (2003) 825-834.

[7] Aracena J., Demongeot J., Goles E., Mathematical modelling in genetic networks, IEEE Trans. Neural Networks 15 (2004) $77-83$.

[8] Aracena J., Demongeot J., Goles E., Fixed points and maximal independent sets on AND-OR networks, Discr. Appl. Math. 138 (2004) 277-288.

[9] Aracena J., Demongeot J., Goles E., On limit-cycles of monotone functions with symmetric connection graphs, Theoret. Comp. Sci. 322 (2004) 237-244.

[10] Françoise J.P., Oscillations en biologie. Analyse qualitative et modèles. Springer, Berlin (2005).

[11] Thom R., Esquisse d'une sémiophysique, Interéditions, Paris (1988).

[12] Demongeot J., Modeling the genetic expression: positive interaction loops and genetic regulatory systems, in: Mathématiques et Biologie, Société Mathématique de France, Paris, 2002, pp. 67-94.

[13] Hilbert D., Sur les problèmes futurs des mathématiques : les 23 problèmes, Jacques Gabay, Paris (1990).

[14] Ben Abdallah N., J. Dolbeault J., Relative Entropies For Kinetic Equations In Bounded Domains (irreversibility, stationary solutions, uniqueness), C. R. Acad. Sci. Paris Sér. I Math. 330 (2000) 867-872.

[15] Tonnelier A., Meignen S., Bosch H., Demongeot J., Synchronization and desynchronization of neural oscillators: comparison of two models, Neural Networks 12 (1999) 1213-1228.

[16] Winfree A.T., The geometry of the biological time, Springer Verlag, Berlin (1980).

[17] Winfree A.T., An integrated view of the resetting of a circadian clock, J. Theor. Biol. 28 (1970) $327-374$.

[18] Kopell N., Ermentrout G.B., On chain of coupled oscillators forced at one end, SIAM J. Appl. Math. 51 (1991) 1397-1417 